\title{
Low Power Consumption Red Light-Emitting Diodes Based on Inorganic Perovskite Quantum Dots under an Alternating Current Driving Mode
}

\author{
Jingjing Liu ${ }^{1,2}$, Zhangbo Lu ${ }^{1,2}$, Xianju Zhang ${ }^{3}$, Yangyi Zhang ${ }^{1,2}$, Haiguang $\mathrm{Ma}^{1}$, Yang $\mathrm{Ji}^{1,2}$, \\ Xiangxing $\mathrm{Xu}^{3}$, Linwei $\mathrm{Yu}^{1}$, Jun $\mathrm{Xu}{ }^{1,2, *}$ and Kunji Chen ${ }^{1}$ \\ 1 National Laboratory of Solid State Microstructures, School of Electronic Science and Engineering and \\ Collaborative Innovation Center of Advanced Microstructures, Nanjing University, Nanjing 210093, China; \\ ljjhenan@163.com (J.L.); luzhangbo123@outlook.com (Z.L.); yyzhan@hotmail.com (Y.Z.); \\ mhgnju@gmail.com (H.M.); Jiyang2018@hotmail.com (Y.J.); yulinwei@nju.edu.cn (L.Y.); \\ kjchen@nju.edu.cn (K.C.) \\ 2 Jiangsu Provincial Key Laboratory of Advanced Photonic and Electronic Materials, Nanjing University, \\ Nanjing 210093, China \\ 3 Jiangsu Key Laboratory of Biofunctional Materials School of Chemistry and Materials Science, Nanjing \\ Normal University, Nanjing 210046, China; Xianjuzhang2018@hotmail.com (X.Z.); xuxx@nju.edu.cn (X.X.) \\ * Correspondence: junxu@nju.edu.cn; Tel.: +86-025-83594836; Fax: +86-025-83595535
}

Received: 31 October 2018; Accepted: 23 November 2018; Published: 26 November 2018

\begin{abstract}
Inorganic perovskites have emerged as a promising candidate for light-emitting devices due to their high stability and tunable band gap. However, the power consumption and brightness have always been an issue for perovskite light-emitting diodes (PeLEDs). Here, we improved the luminescence intensity and decreased the current density of the PeLEDs based on $\mathrm{CsPbI}_{3}$ quantum dots (QDs) and p-type Si substrate through an alternating current (AC) driving mode. For the different driving voltage modes (under a sine pulsed bias or square pulsed bias), a frequency-dependent electroluminescent (EL) behavior was observed. The devices under a square pulsed bias present a stronger EL intensity under the same voltage due to less thermal degradation at the interface. The red PeLEDs under a square pulsed bias driving demonstrate that the EL intensity drop-off phenomenon was further improved, and the integrated EL intensity shows the almost linear increase with the increasing driving voltage above $8.5 \mathrm{~V}$. Additionally, compared to the direct current (DC) driving mode, the red PeLEDs under the AC condition exhibit higher operating stability, which is mainly due to the reducing accumulated charges in the devices. Our work provides an effective approach for obtaining strong brightness, low power consumption, and high stability light-emitting devices, which will exert a profound influence on coupling LEDs with household power supplies directly.
\end{abstract}

Keywords: perovskite quantum dots; light emitting diodes; alternating current driving; low power consumption; silicon

\section{Introduction}

Perovskite materials are emerging as semiconductors with potential applications in optoelectronic devices [1-8]. Their characteristics of high color purity [9-12], tunable band gap [13-17], and low cost $[18,19]$ are favorable for obtaining light-emitting diodes (LEDs). However, the poor operating stability of perovskite light-emitting diodes (PeLEDs) directly obstructs commercial applications, especially for red light emitting devices. For the perovskite materials, the air-exposure stability of the inorganic perovskite $\left(\mathrm{CsPbX}_{3}, \mathrm{X}=\mathrm{Cl}, \mathrm{Br}\right.$, I) is superior to organic perovskite $\left(\mathrm{CH}_{3} \mathrm{NH}_{3} \mathrm{PbX}_{3}, \mathrm{X}=\mathrm{Cl}\right.$, $\mathrm{Br}, \mathrm{I})$ as a result of replacing methyl ammonium with Cs cations [20-22]. This is crucial for practical 
optoelectronic applications, where long-term stability and cost effectiveness are vital. In previous works [23-26], the PeLEDs were usually studied under a direct current (DC) driving condition and they have one thing in common: the electroluminescent (EL) intensity drops off obviously at a high driving voltage due to heat generation and charge accumulation in the defect states, which would increase the power consumption of the devices. As a consequence, it is necessary to suppress heat generation and reduce charge accumulation in the defect states to obtain low power consumption devices.

EL devices operating under alternating current (AC) electricity have attracted more and more attention: one reason is the unique light-emitting mechanism of carrier generation and recombination; the other important one is their great potential application in sensors, lighting, and displays [27-30]. The AC condition has the advantage of reversal voltage, avoiding more charge accumulation at the device's interface and a short operating time resulting in less heat generation compared to DC mode. Moreover, the trapped charge can be extracted in each period resulting in less defect creation [31]. In our previous work, the device performance based on $\mathrm{Si}$ quantum dots (QDs) $/ \mathrm{SiO}_{2}$ multilayers [31] and green PeLEDs [32] under the AC driving mode was studied, respectively. A high operating stability was obtained for the two different devices due to less thermal degradation of the device induced by less operation time.

In this work, an optimized multilayered structure of $\mathrm{Al} / \mathrm{p}-\mathrm{Si} / \mathrm{Poly}-\mathrm{TPD} / \mathrm{CsPbI} / \mathrm{ZnO} / \mathrm{ITO}$ was created as the PeLEDs. In order to improve luminescence intensity and reduce the power consumption, the luminescence and electrical properties of the devices under the sine pulsed bias and square pulsed bias driving modes, and DC driving mode, were studied systematically. For devices under different AC driving voltage modes (sine pulsed bias and square pulsed bias), frequency-dependent electroluminescent behavior was observed. The red PeLEDs under square pulsed bias driving demonstrates that the EL intensity drop-off phenomenon was further improved. The EL intensity was also further enhanced, and the current density was decreased at the same driving voltage. Our findings provide an effective approach for obtaining a strong brightness, low power consumption, and high stability light-emitting device, which will exert profound influence on directly coupled LEDs with household power supplies.

\section{Experimental Details}

We purchased $\mathrm{PbI}_{2}, \mathrm{Cs}_{2} \mathrm{CO}_{3}, \mathrm{ZnCl}_{2}$, oleylamine, and cyclohexane from Aladdln Industrial Corporation (Shanghai, China) and purchased 1-octadecene and oleic acid from Alfa Aesar (Shanghai, China). The experimental details of synthesis of $\mathrm{CsPbI}_{3}$ QDs can be found in our previous work [32,33]. Direct centrifugation was used to separate the $\mathrm{CsPbI}_{3}$ QDs from the reaction solution. The precipitated $\mathrm{CsPbI}_{3}$ QDs were re-dispersed in cyclohexane.

The LEDs were fabricated on p-type Si wafers. The Si wafer was firstly cleaned with a mixture of hydrogen peroxide and ammonium hydroxide, then cleaned with a mixture of hydrogen peroxide and hydrochloride acid. Lastly, the Si wafer was cleaned with deionized water and dried by nitrogen. An aluminum electrode was deposited by thermal evaporation. The concentration of poly-TPD was $4 \mathrm{mg} \mathrm{mL}^{-1}$ and was spin-coated onto the p-Si substrate at $4000 \mathrm{rpm}$ for $60 \mathrm{~s}$. The concentration of $\mathrm{CsPbI}_{3}$ QDs was $10 \mathrm{mg} \mathrm{mL}^{-1}$ and was spin-coated onto the poly-TPD layer at $2000 \mathrm{rpm}$ for $60 \mathrm{~s}$. ZnO was deposited using a magnetron sputtering system. The ITO electrode was a circle with a diameter of $1.5 \mathrm{~mm}$, which was deposited by the magnetron sputtering system through a circle shadow mask.

The microstructures of $\mathrm{CsPbI}_{3}$ QDs were characterized by transmission electron microscopy (TEM, TecnaiG2F20, Hillsboro, OR, USA) and the structure of $\mathrm{CsPbI}_{3}$ QDs were characterized by $\mathrm{X}$-ray powder diffractometer (XRD, Rigaku, Akishima, Tokyo, Japan). Field emission scanning electron microscopy (FESEM, Sigma, Oberkochen, Germany) was employed to characterize the cross-sectional morphologies of the device. Optical absorption spectra of $\mathrm{CsPbI}_{3}$ QDs were obtained through a Ultraviolet-Visible-Near Infrared (UV-VIS-NIR) spectrometer (UV 3600, Shimadzu, Kyoto, Japan). A HORIBA Jobin Yvon system with a synapse (photomultiplier tube) PMT detector was utilized to 
obtain the photoluminescence spectra and the EL signals under both AC and DC bias. All the tests were conducted under room temperature.

\section{Results and Discussion}

Figure 1a presents the transmission electron microscopy (TEM) image of $\mathrm{CsPbI}_{3}$ QDs, in which the monodisperse size distribution of QDs can be identified. The histogram of the size distribution of the $\mathrm{CsPbI}_{3}$ QDs demonstrates that $\mathrm{CsPbI}_{3}$ QDs have an average diameter of $\sim 13 \mathrm{~nm}$, and the size distribution range from 11 to $15 \mathrm{~nm}$ are shown in Figure 1b. In the high-resolution TEM (HRTEM) image shown in Figure 1c, the crystal lattice is clearly resolved with the lattice spacing of $0.62 \mathrm{~nm}$, which corresponds to the (100) crystallographic plane of $\mathrm{CsPbI}_{3}[11,34]$. The XRD pattern of $\mathrm{CsPbI}_{3}$ QDs is displayed in Figure 1d. The characteristic diffraction peaks at $13.92^{\circ}, 20.17^{\circ}, 24.53^{\circ}, 28.33^{\circ}$, $31.67^{\circ}, 35.27^{\circ}$, and $40.82^{\circ}$ can be assigned to the diffractions from (100), (110), (111), (200), (210), (211), and (220) crystal planes, respectively. These results demonstrate that inorganic perovskite quantum dots (IPQDs) can be attested to the bulk cubic $\mathrm{CsPbI}_{3}$ with high crystalline quality. Figure 1e shows the absorption and photoluminescence (PL) spectra of $\mathrm{CsPbI}_{3}$ QDs. The absorption peak of $\mathrm{CsPbI}$ QDs is obvious and strong. Absorption onset occurs at $\sim 702 \mathrm{~nm}$ for $\mathrm{CsPbI}_{3}$ QDs and a strong PL peak of $\mathrm{CsPbI}_{3}$ QDs is located at $685 \mathrm{~nm}$. The full width at half maximum (FWHM) of the PL band is as narrow as $30 \mathrm{~nm}$, which is helpful to obtain a high color purity.
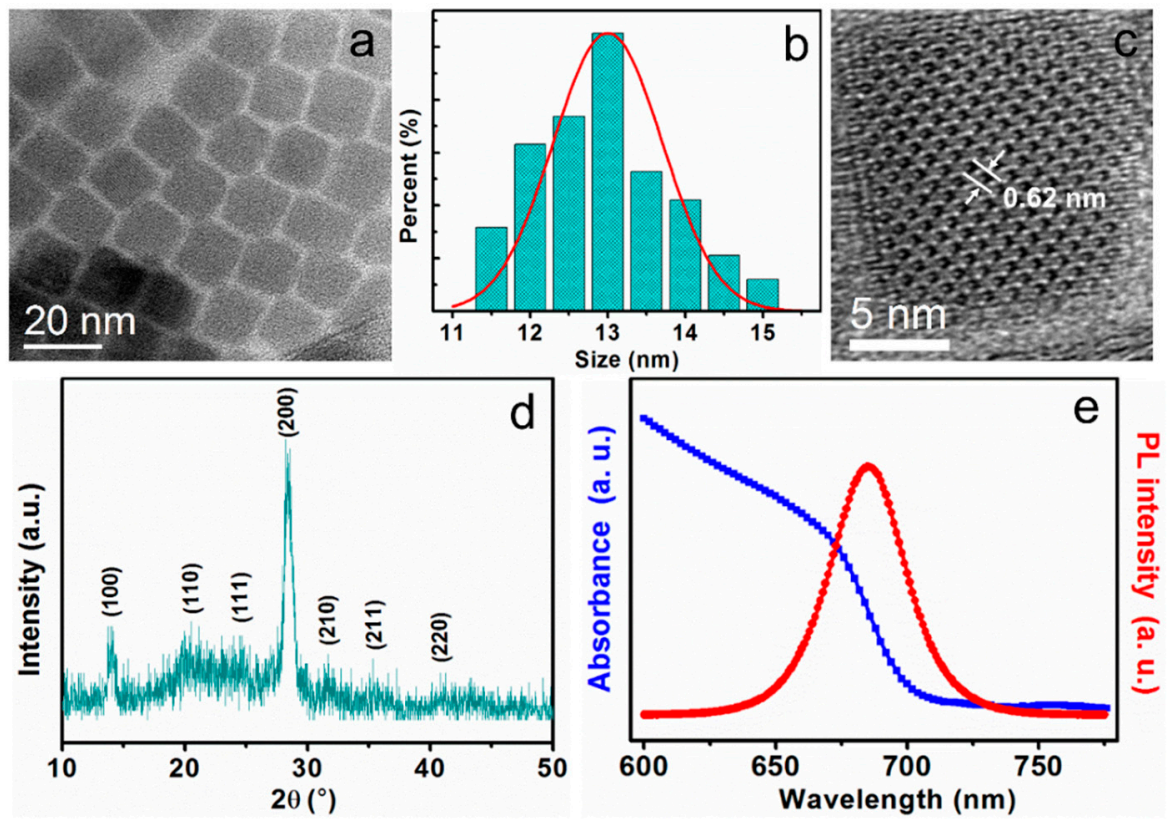

Figure 1. (a) TEM image of monodisperse $\mathrm{CsPbI}_{3}$ quantum dots (QDs); (b) Histogram for the size distribution of the $\mathrm{CsPbI}_{3}$ QDs; (c) High-resolution TEM (HRTEM) image of monodisperse $\mathrm{CsPbI}_{3}$ QDs; (d) The XRD spectrum of $\mathrm{CsPbI}_{3}$ QDs; (e) UV-vis absorption and photoluminescence (PL) spectra (with $325 \mathrm{~nm}$ excitation wavelength) of $\mathrm{CsPbI}_{3}$ QDs.

PeLEDs were fabricated with a structure of ITO/ZnO/CsPbI 3 QDs/poly-TPD/p-Si/Al (ITO: indium tin oxide, poly-TPD: poly [N, $\mathrm{N}^{\prime}$-bis (4-butylphenyl)- $\mathrm{N}, \mathrm{N}^{\prime}$-bis (phenyl)-benzidine]), shown schematically in Figure 2a. Figure 2b presents cross-sectional SEM images of a complete PeLED. The thickness of the poly-TPD layer is about $10 \mathrm{~nm}$ and the $\mathrm{CsPbI}_{3}$ QDs layer is about $30 \mathrm{~nm}$. The thickness of the $\mathrm{ZnO}$ and ITO film is about 45 and $230 \mathrm{~nm}$, respectively. Figure 2c shows a schematic energy diagram of the device. Further, Al was used as anode, p-Si was used as a hole injection layer to decrease the hole barrier for efficient hole injection. Poly-TPD was used as hole transport and electron blocking layers, and $\mathrm{CsPbI}_{3}$ QDs were the emissive layer. $\mathrm{ZnO}$ was used as 
electron transport and hole blocking layers. ITO was utilized as a cathode. Light emission occurs when injected holes and electrons meet in the $\mathrm{CsPbI}_{3}$ QDs layer and recombine radiatively.

a

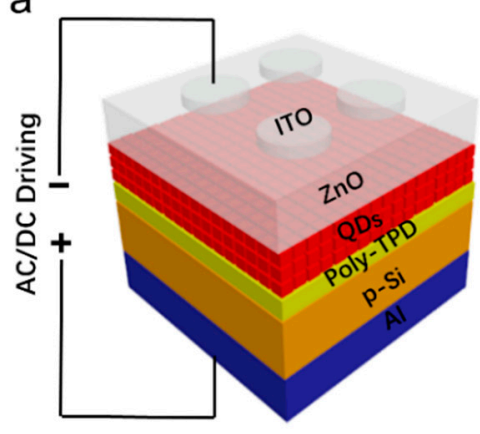

b

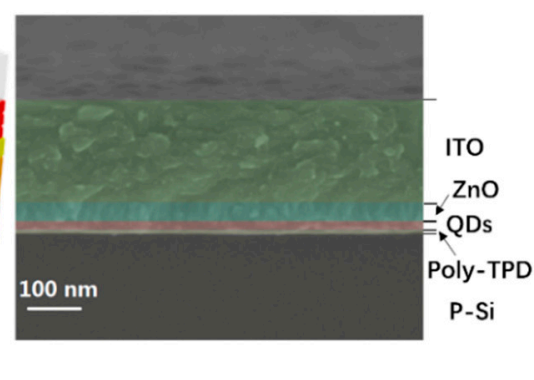

C

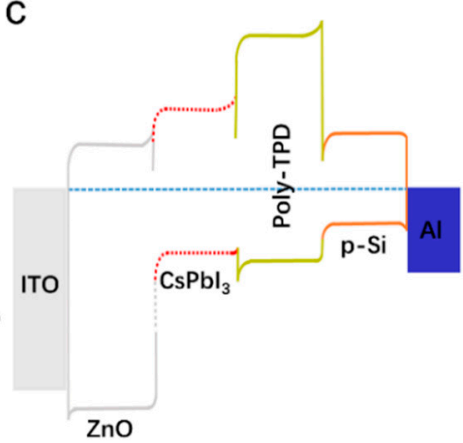

Figure 2. (a) Schematic structure of perovskite light-emitting diodes (PeLEDs); (b) SEM image of the cross section of PeLEDs; (c) Energy band diagrams of PeLEDs. AC: alternating current; DC: direct current.

Figure 3a shows the current density-voltage $(\mathrm{J}-\mathrm{V})$ characteristics of the devices under AC and DC driving modes. The schematic diagram of the sine pulsed bias used in PeLEDs for AC driving is displayed in the inset of Figure 3a. The sine pulsed bias possesses the influence factors of Vpp, frequency, and duty cycle. For the sine pulsed mode, the driving Vpp ranges from 4 to $10 \mathrm{~V}$, the duty cycle is $50 \%$, and the excitation frequency is $10 \mathrm{~Hz}$. When the device is driven under the DC mode, the driving voltage ranges from 4 to $10 \mathrm{~V}$, which corresponds with AC driving Vpp. In previous work [33], the $\mathrm{CsPbI}_{3}$ QDs LEDs present current difference under the various bias polarizations due to the p-n heterojunction being formed in $\mathrm{CsPbI}_{3}$ QDs LEDs. As a result, the devices can only function under forward bias. For sine pulsed bias, the maximum forward voltage is equal to $\mathrm{Vpp} / 2$, as shown in the inset of Figure 3a. As exhibited in Figure 3a, the current density under AC bias is obviously lower than that of DC due to the working voltage under AC driving mode being lower than that under DC driving mode. Figure $3 \mathrm{~b}$ shows the integrated EL intensity as a function of applied voltage under both the AC (sine pulsed bias) and DC driving conditions. The sine pulsed bias in Figure $3 \mathrm{~b}$ presents $10 \mathrm{~Hz}$ frequency and 50\% duty cycle. The integrated EL intensity under DC bias is greater than that under $\mathrm{AC}$ bias when the driving voltage is lower than $7 \mathrm{~V}$. While under high driving voltage, the integrated EL intensity under DC bias becomes lower than that under AC bias. Furthermore, the integrated EL intensity shows a decreasing tendency when the DC driving voltage higher than $8.5 \mathrm{~V}$. It is obvious that the EL intensity drop-off phenomenon under high voltage is greatly improved under the $\mathrm{AC}$ driving mode compared to the devices under the $\mathrm{DC}$ driving mode. Figure $3 \mathrm{c}$ shows the EL spectra of $\mathrm{CsPbI}_{3}$ QDs LEDs under DC (8 V) and AC driving modes (sine pulsed bias $8 \mathrm{~V} \mathrm{Vpp).}$ It was found that the EL intensity under AC (sine pulsed bias) driving mode is improved by $26 \%$ than the DC driving one. Obviously, the devices working under an AC bias have the advantage of low power consumption. The improvement in light emission performance under AC drive mode can be attributed to suppressed heat generation at a high current density due to the short running time of the device.

In order to study device performance under different AC modes, we also apply the square pulsed bias on $\mathrm{CsPbI}_{3}$ QDs LEDs. Figure 4a shows the integrated EL intensity against the excitation frequency (from $10 \mathrm{~Hz}$ to $10 \mathrm{kHz}$ ) under the square pulsed bias with $50 \%$ duty cycle and different driving voltage (Vpp from 8 to $10 \mathrm{~V}$ ). With increasing driving voltage, the integrated EL intensity becomes stronger under the same excitation frequency. The light emission is frequency dependent in our devices and the integrated EL intensity from all devices decreases as the frequency increases. When the excitation frequency is lower than $100 \mathrm{~Hz}$, integrated EL intensity changes gently. A stable and bright red-light emission is obtained when the $\mathrm{CsPbI}_{3}$ QDs LEDs are driven under a square pulsed bias with $8 \mathrm{~V} \mathrm{Vpp}$ and $50 \mathrm{~Hz}$ excitation frequency. It demonstrates that AC driving LEDs 
can be used directly for household power supplies. The inset in Figure $4 \mathrm{a}$ is the schematic diagram of a square pulsed bias which possesses the same influence factors as a sine pulsed bias. Figure $4 \mathrm{~b}$ is the J-V relationship of $\mathrm{CsPbI}_{3}$ QDs LEDs under the AC (square pulsed bias) driving condition. With increasing excitation frequency from $10 \mathrm{~Hz}$ to $10 \mathrm{kHz}$, the effective current density of $\mathrm{CsPbI}_{3}$ QDs LEDs decreased, which resulted in the integrated EL intensity decay. When the frequency rises above $\sim 10 \mathrm{kHz}$ with increasing excitation frequency, more and more injected carriers will be electrically pumped out before they can recombine with the latter ones. Thus, the EL intensity and current density all drop with an increase of frequency. The $\mathrm{CsPbI}_{3}$ QDs LEDs show sharp EL peaks under various biases, as shown in Figure 4c, with peak wavelengths of $685 \mathrm{~nm}$ and a narrow FWHM of $\sim 30 \mathrm{~nm}$. The inset in Figure 4c shows the EL spectrum of the $\mathrm{CsPbI}_{3}$ QDs LEDs under $4 \mathrm{~V}$ Vpp which also presents a strong luminescence peak.
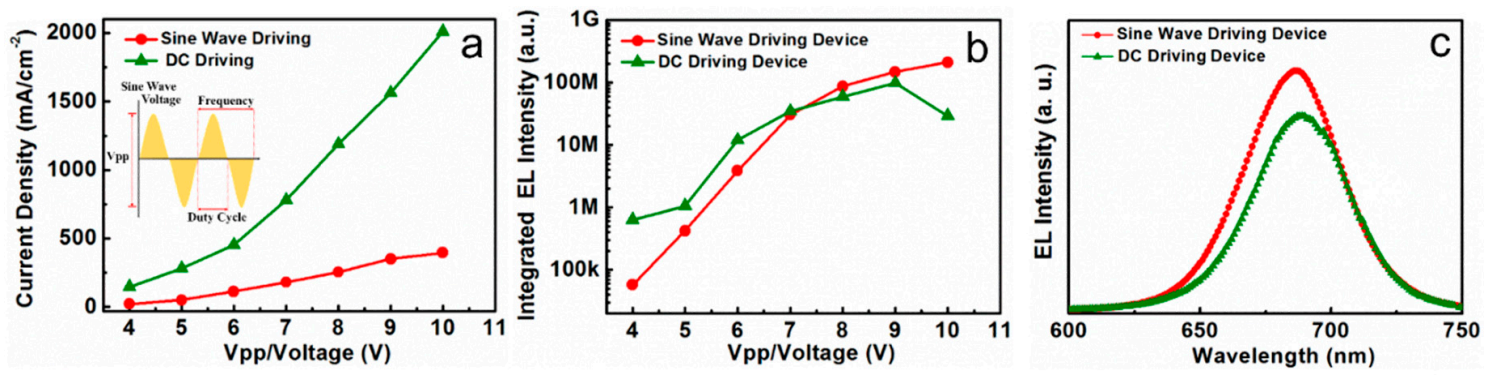

Figure 3. (a) Density-voltage curve of the $\mathrm{CsPbI}_{3}$ QDs light-emitting diodes (LEDs) under DC and AC (sine pulsed bias, duty cycle: $50 \%$, frequency: $10 \mathrm{~Hz}$ ) driving conditions. The inset is a schematic diagram of the sine pulsed bias; (b) Integrated electroluminescent (EL) intensity of $\mathrm{CsPbI}_{3}$ QDs LEDs versus voltage under DC and AC driving conditions (sine pulsed bias, duty cycle: $50 \%$, frequency: $10 \mathrm{~Hz}$ ); (c) The EL intensity of devices under DC (8 V) and AC (sine pulsed bias, duty cycle: 50\%, frequency: $10 \mathrm{~Hz}, \mathrm{Vpp}: 8 \mathrm{~V})$ driving modes.
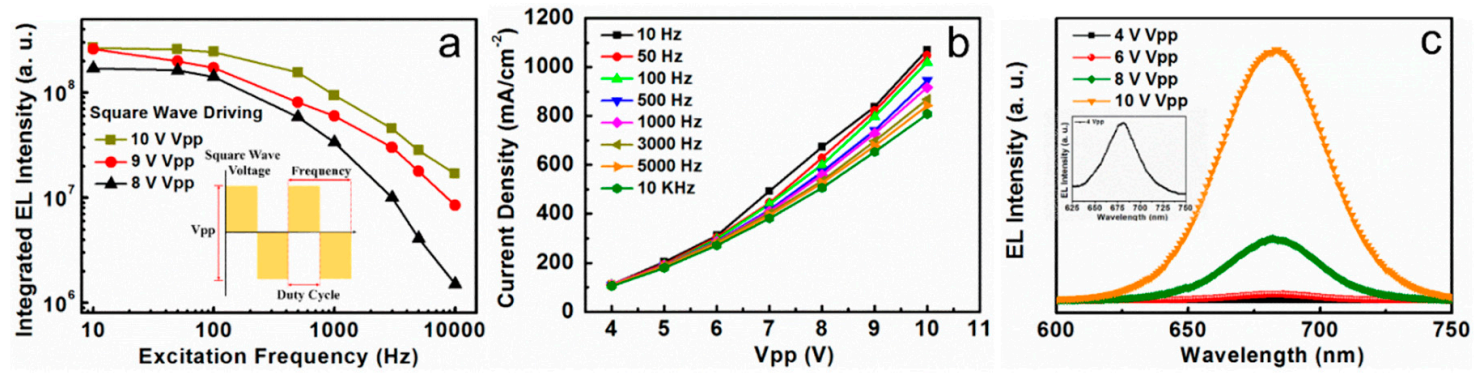

Figure 4. (a) The integrated EL intensity versus excitation frequency (from $10 \mathrm{~Hz}$ to $10 \mathrm{kHz}$ ) under different driving voltages with $50 \%$ duty cycle square pulsed bias. Illustration is a schematic diagram of square wave waveform; (b) Current density-voltage (J-V) characteristics of devices under different excitation frequency with 50\% duty cycle square pulsed bias; (c) The EL intensity versus Vpp (from 4 to $10 \mathrm{~V}$ ) with $10 \mathrm{~Hz}$ excitation frequency and 50\% duty cycle under square pulsed bias. The inset shows the EL spectrum of the $\mathrm{CsPbI}_{3}$ QDs LEDs under $4 \mathrm{~V}$ Vpp.

The integrated EL intensity against the applied voltage under different driving modes (square pulsed bias and sine pulsed bias) is plotted in Figure 5a. The integrated EL intensity is gradually increased by increasing the driving voltage in all devices at a fixed frequency of $10 \mathrm{~Hz}$ and $50 \%$ duty cycle. For the different driving voltage modes (square pulsed bias and sine pulsed bias), the integrated EL intensity presents a similar changing trend. Furthermore, we noticed the integrated EL intensity under the square pulsed bias is stronger than that under the sine pulsed bias driving condition (Vpp from 4 to $10 \mathrm{~V}$ ). When the driving $\mathrm{Vpp}$ is $10 \mathrm{~V}$, the integrated EL intensity under the square pulsed bias is about twice as strong than that under the sine pulsed bias. There is often an emission intensity drop-off phenomenon of PeLEDs under high voltage for the DC driving mode $[23,35]$. The emission 
density under the AC driving mode obtains a significant improvement in the drop-off phenomenon. Figure $5 \mathrm{~b}$ shows the working current density of the devices under different driving Vpp. For the two different driving voltage modes, the driving Vpp ranges from 4 to $10 \mathrm{~V}$, the duty cycle and the excitation frequency is $50 \%$ and $10 \mathrm{~Hz}$, respectively. Figure $5 \mathrm{~b}$ exhibits high current flowing in the devices at a high driving voltage. As discussed above, the devices can only function under forward bias. As shown in the inset of Figure 4a, the working voltage of the square pulsed bias is equal to Vpp/2. Obviously, the working voltage under the square pulsed bias is higher than that under the sine pulsed bias at the same Vpp. As a result, the current under the square pulsed bias is higher than that under the sine pulsed bias, which would result in stronger emission intensity. The results are in good agreement with the observed results in Figure 5a. Figure $5 \mathrm{c}$ displays the influence of excitation frequency on the EL intensity. The excitation frequency ranges from $10 \mathrm{~Hz}$ to $10 \mathrm{kHz}$ with a $10 \mathrm{~V}$ driving voltage and $50 \%$ duty cycle under a sine pulsed bias and a square pulsed bias. With increasing excitation frequency, all integrated EL intensity declined. The integrated EL intensity under the square pulsed bias is obviously stronger than that under the sine pulsed bias. As shown in Figure $5 \mathrm{~d}$, all currents are declining with an increasing excitation frequency from $10 \mathrm{~Hz}$ to $10 \mathrm{kHz}$, due to more and more injected carriers that will be electrically pumped out before they can recombine with the latter ones. The square pulsed current is a little higher than the sine pulsed current, because the working voltage under the square pulsed bias is higher than that under the sine pulsed bias at the same Vpp. The $\mathrm{CsPbI}_{3}$ QDs LEDs under the square pulsed bias present superior performance.
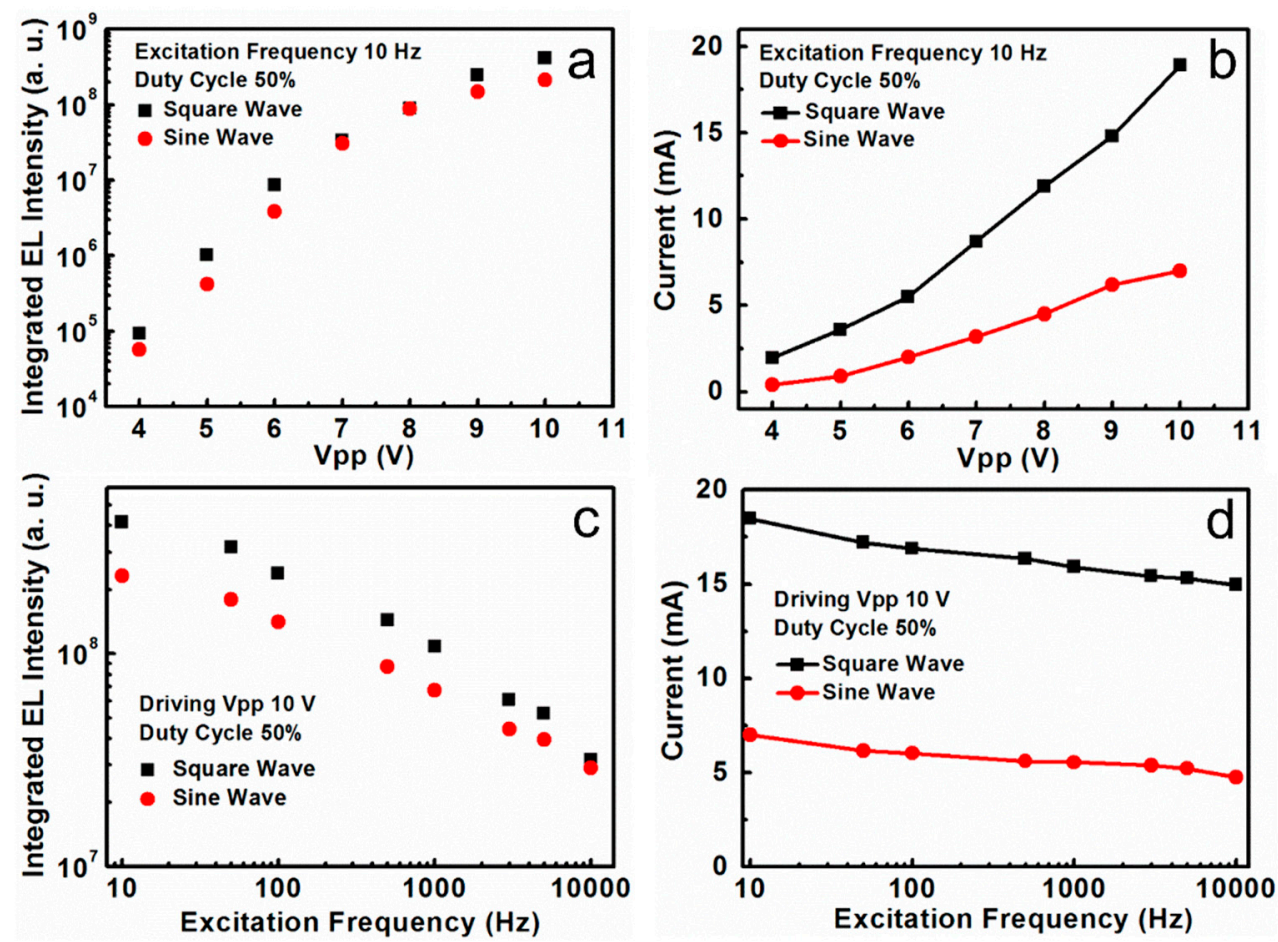

Figure 5. (a) The integrated EL intensity versus Vpp (from 4 to $10 \mathrm{~V}$ ) with $10 \mathrm{~Hz}$ excitation frequency and $50 \%$ duty cycle under different driving voltage modes (square wave and sine wave); (b) Current versus Vpp for the devices under square pulsed bias and sine pulsed bias with $10 \mathrm{~Hz}$ excitation frequency and 50\% duty cycle; (c) The integrated EL intensity versus excitation frequency (from $10 \mathrm{~Hz}$ to $10 \mathrm{kHz}$ ) with $10 \mathrm{~V}$ driving voltage and $50 \%$ duty cycle under different driving voltage modes (square pulsed bias and sine pulsed bias); (d) Current versus excitation frequency for the devices under square pulsed bias and sine pulsed bias with $10 \mathrm{~V} \mathrm{Vpp}$ and 50\% duty cycle.

The effective current density under the square pulsed bias is obviously lower than that of the DC bias, as shown in Figure 6a, which demonstrates the devices working under AC bias have the advantage of low power consumption. The integrated EL intensities are plotted in Figure $6 \mathrm{~b}$ as a 
function of applied bias under both the AC (square wave bias) and DC driving modes. The integrated EL intensity drops off after applying a DC bias higher than $8.5 \mathrm{~V}$, but the integrated EL intensity under the square pulsed bias increases almost linearly with increasing driving voltage. The EL intensity drop-off phenomenon is further improved by square wave bias driving. Figure 6c shows the EL spectra of $\mathrm{CsPbI}_{3}$ QDs LEDs under DC and AC (square wave bias) driving modes (8 V bias). All the peaks of the devices were equipped with $30 \mathrm{~nm}$ FWHM. It was noticed that the EL intensity under the square wave bias was improved by $33 \%$ than that under DC driving. The insets are the digital pictures showing the red-light emission from devices under $\mathrm{AC}$ and DC driving conditions. In our previous work [33], the brightness of the $\mathrm{CsPbI}_{3}$ QDs LEDs under the DC driving condition reach the maximum value of $665 \mathrm{~cd} \mathrm{~m}^{-2}$. The brightness of the $\mathrm{CsPbI}_{3}$ QDs LEDs under the AC driving condition is about $850 \mathrm{~cd} \mathrm{~m}^{-2}$ at $8 \mathrm{~V}$, which is estimated according to the integrated EL intensity. The enhanced luminescence performance under the AC driving condition can be attributed to the suppressed charge accumulation. In view of $\mathrm{CsPbI}_{3}$ QDs LEDs only working under a positive voltage bias because of the existing p-n heterojunction [33], the negative voltage bias of the AC mode will extract the trapped carriers, and then avoid more charge accumulation than that of the DC mode in the device.
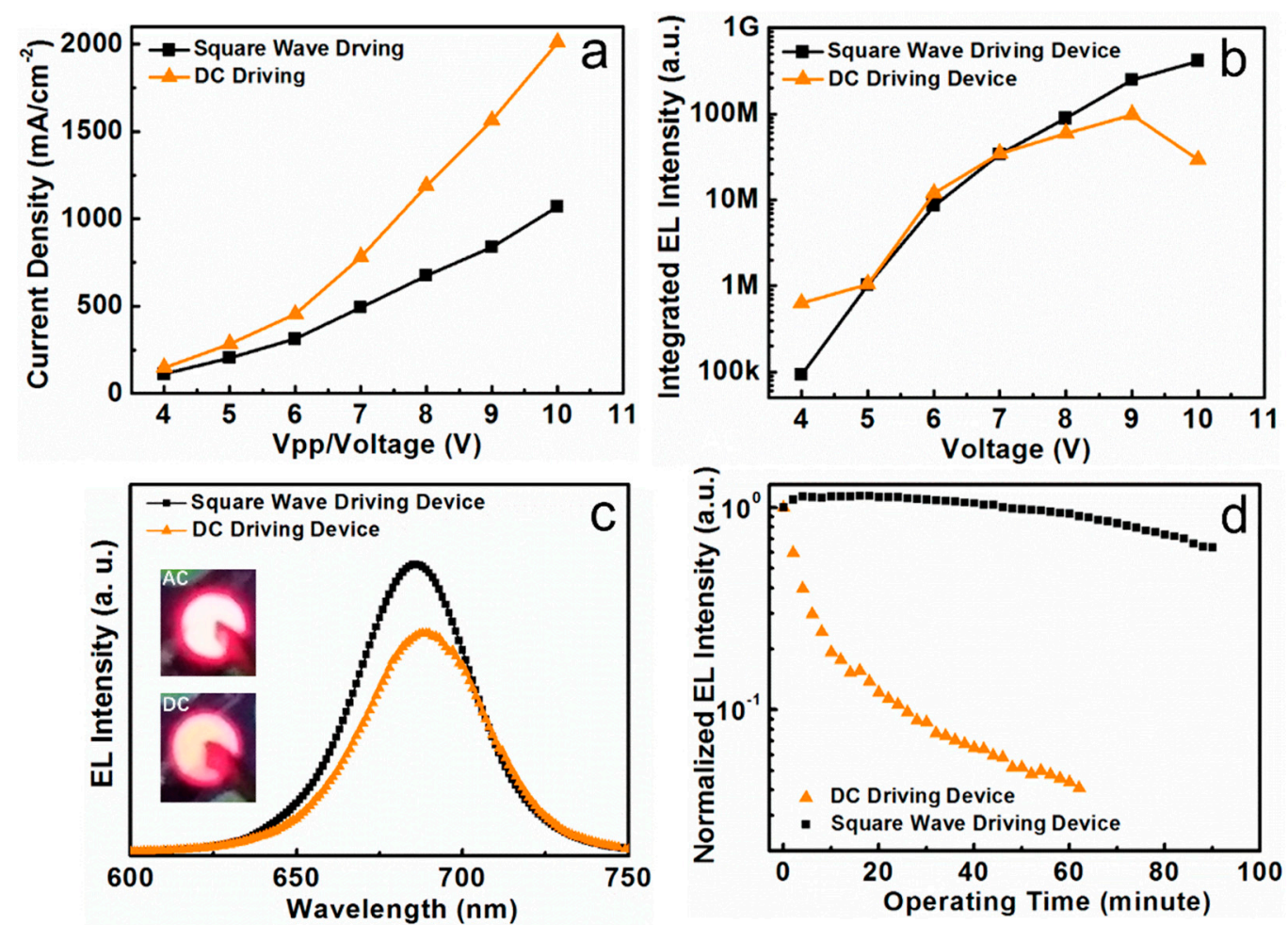

Figure 6. (a) Density-voltage curve of the $\mathrm{CsPbI}_{3}$ QDs LEDs under DC and AC (square pulsed bias, duty cycle: $50 \%$, frequency: $10 \mathrm{~Hz}$ ) driving conditions; (b) Integrated EL intensity of $\mathrm{CsPbI}_{3}$ QDs LEDs with different voltages under DC and AC driving conditions (square pulsed bias, duty cycle: 50\%, frequency: $10 \mathrm{~Hz}$ ); (c) The EL intensity of devices under DC (8 V) and AC (square pulsed bias, duty cycle: $50 \%$, frequency: $10 \mathrm{~Hz}, \mathrm{Vpp}: 8 \mathrm{~V}$ ) driving conditions. The insets are the digital pictures showing the red-light emission from devices under AC and DC driving conditions; (d) Normalized emission decays of the PeLEDs after different running periods under DC $(8 \mathrm{~V})$ and AC (square pulsed bias, duty cycle: $50 \%$, frequency: $10 \mathrm{~Hz}$, Vpp: $8 \mathrm{~V}$ ) driving conditions.

In order to better understand the effect of driving modes on the PeLEDs, long-term operation tests in an ambient environment was further performed. The operation stability of devices under DC bias and AC (square pulsed) bias is shown in Figure 6d. We obtained the EL intensity decay variation of unpackaged devices in an atmospheric environment. During the test, a continuous $8 \mathrm{~V} / 8 \mathrm{~V}$ Vpp voltage is injected and the EL intensity is recorded every two minutes. Under the DC driving 
mode, there is a $96 \%$ emission decay after $60 \mathrm{~min}$. However, there is only a $36 \%$ emission decay after $90 \mathrm{~min}$ of operating time under the AC driving mode and the stability of the device is improved significantly. The operational stability under the AC driving mode is superior to PeLEDs under the DC driving condition, which can be attributed to less thermal degradation induced by the shorter operation time under the frequent reverse of applied bias. Also, the current density was smaller than that under the DC driving mode with the same applied voltage, which is also helpful for reducing power consumption and improving the device endurance.

\section{Conclusions}

We designed and fabricated LEDs that combined the inorganic perovskite $\mathrm{CsPbI}_{3}$ QDs with the p-type Si substrate. The luminescence and electrical properties of the devices under the AC driving mode and the DC driving mode were studied systematically. The frequency-dependent EL behavior was observed. With increasing excitation frequency, the emission density decreased. For the different AC driving voltage modes (square pulsed bias and sine pulsed bias), the integrated EL intensity of devices under the square pulsed bias was stronger than that under the sine pulsed bias due to the higher injection current. The resulting red PeLEDs under AC driving demonstrate a relatively low power consumption, and the EL intensity drop-off phenomenon is obviously improved compared to that under DC driving. The decay of emission intensity is less than $36 \%$ after 90 min for the AC driving condition, which demonstrates that the PeLEDs exhibit higher operating stability compared to the DC driving one due to the reduced accumulated charges in the devices. It is greatly significant to study the luminescence and electrical properties under the AC driving mode by applying different mode pulsed biases. The findings will exert a profound influence on directly coupled LEDs with household power supplies.

Author Contributions: J.L: Device preparation, performance testing, data curation, formal analysis, writing-original draft; Z.L.: Preparing ZnO films; X.Z.: Synthesising $\mathrm{CsPbI}_{3}$ QDs; Y.Z.: Providing XRD data of $\mathrm{CsPbI}_{3}$ QDs; H.M.: Providing SEM data of device; Y.J.: Providing TEM data of $\mathrm{CsPbI}_{3}$ QDs; X.X.: Project administration, resources; L.Y.: Supervision; J.X.: Project administration, resources, supervision; K.C.: Supervision.

Funding: The authors acknowledge the financial support from the National Natural Science Foundation of China (NSFC) under Nos No. 61735008, 51572120, and 11774155, Priority Academic Program Development of Jiangsu Higher Education Institutions (PAPD).

Conflicts of Interest: The authors declare no conflict of interest.

\section{References}

1. Xing, G.; Mathews, N.; Lim, S.S.; Yantara, N.; Liu, X.; Sabba, D.; Grätzel, M.; Mhaisalkar, S.; Sum, T.C. Low-Temperature Solution-Processed Wavelength-Tunable Perovskites for Lasing. Nat. Mater. 2014, 13, 476-480. [CrossRef] [PubMed]

2. Stranks, S.D.; Snaith, H.J. Metal-Halide Perovskites for Photovoltaic and Light-Emitting Devices. Nat. Nanotechnol. 2015, 10, 391-402. [CrossRef] [PubMed]

3. Zhao, Y.; Zhu, K. Organic-Inorganic Hybrid Lead Halide Perovskites for Optoelectronic and Electronic Applications. Chem. Soc. Rev. 2016, 45, 655-689. [CrossRef] [PubMed]

4. Docampo, P.; Bein, T. A Long-Term View on Perovskite Optoelectronics. Acc. Chem. Res. 2016, 49, $339-346$. [CrossRef] [PubMed]

5. Tsai, H.; Nie, W.; Lin, Y.; Blancon, J.C.; Tretiak, S.; Even, J.; Gupta, G.; Ajayan, P.M.; Mohite, A.D. Effect of Precursor Solution Aging on the Crystallinity and Photovoltaic Performance of Perovskite Solar Cells. Adv. Energy Mater. 2017, 7, 1602159. [CrossRef]

6. Sargent, B.R.S.A. Perovskite Photonic Sources. Nat. Photonics 2016, 10, 295-302.

7. Era, M.; Morimoto, S.; Tsutsui, T.; Saito, S. Organic-inorganic Heterostructure Electroluminescent Device Using A Layered Perovskite Semiconductor $\left(\mathrm{C}_{6} \mathrm{H}_{5} \mathrm{C}_{2} \mathrm{H}_{4} \mathrm{NH}_{3}\right)_{2} \mathrm{PbI}_{4}$. Appl. Phys. Lett. 1994, 65, 676-678. [CrossRef] 
8. Koutselas, I.; Bampoulis, P.; Maratou, E.; Evagelinou, T.; Pagona, G.; Papavassiliou, G.C. Some Unconventional Organic Inorganic Hybrid Low-Dimensional Semiconductors and Related Light-Emitting Devices. J. Phys. Chem. C 2011, 115, 8475-8483. [CrossRef]

9. Liang, D.; Peng, Y.; Fu, Y.; Shearer, M.J.; Zhang, J.; Zhai, J.; Zhang, Y.; Hamers, R.J.; Andrew, T.L.; Jin, S. Color-Pure Violet-Light-Emitting Diodes Based on Layered Lead Halide Perovskite Nanoplates. ACS Nano 2016, 10, 6897-6904. [CrossRef] [PubMed]

10. Xing, J.; Yan, F.; Zhao, Y.; Chen, S.; Yu, H.; Zhang, Q.; Zeng, R.; Demir, H.V.; Sun, X.; Huan, A.; et al. High-Efficiency Light-Emitting Diodes of Organometal Halide Perovskite Amorphous Nanoparticles. ACS Nano 2016, 10, 6623-6630. [CrossRef] [PubMed]

11. Kim, Y.; Wolf, C.; Kim, Y.; Cho, H.; Kwon, W.; Do, S.; Sadhanala, A.; Park, C.G.; Rhee, S.; Im, S.H.; et al. Highly Efficient Light-Emitting Diodes of Colloidal Metal-Halide Perovskite Nanocrystals beyond Quantum Size. ACS Nano 2017, 11, 6586-6593. [CrossRef] [PubMed]

12. Kim, Y.; Cho, H.; Lee, T. Metal Halide Perovskite Light Emitters. Proc. Natl. Acad. Sci. USA 2016, 113, 11694-11702. [CrossRef] [PubMed]

13. Tsai, H.; Nie, W.; Blancon, J.C.; Stoumpos, C.C.; Soe, C.M.M.; Yoo, J.; Crochet, J.; Tretiak, S.; Even, J.; Sadhanala, A.; et al. Stable Light-Emitting Diodes Using Phase-Pure Ruddlesden-Popper Layered Perovskites. Adv. Mater. 2018, 30, 1704217. [CrossRef] [PubMed]

14. Levchuk, I.; Osvet, A.; Tang, X.; Brandl, M.; Perea, J.D.; Hoegl, F.; Matt, G.J.; Hock, R.; Batentschuk, M.; Brabec, C.J. Brightly Luminescent and Color-Tunable Formamidinium Lead Halide Perovskite FAPbX 3 $(\mathrm{X}=\mathrm{Cl}, \mathrm{Br}$ I) Colloidal Nanocrystals. Nano Lett. 2017, 17, 2765-2770. [CrossRef] [PubMed]

15. Yuan, M.; Quan, L.N.; Comin, R.; Walters, G.; Sabatini, R.; Voznyy, O.; Hoogland, S.; Zhao, Y.; Beauregard, E.M.; Kanjanaboos, P.; et al. Perovskite Energy Funnels for Efficient Light-Emitting Diodes. Nat. Nanotechnol. 2016, 11, 872-877. [CrossRef] [PubMed]

16. Akkerman, Q.A.; Innocenzo, V.D.; Accornero, S.; Scarpellini, A.; Petrozza, A.; Prato, M.; Manna, L. Tuning the Optical Properties of Cesium Lead Halide Perovskite Nanocrystals by Anion Exchange Reactions. J. Am. Chem. Soc. 2015, 137, 10276-10281. [CrossRef] [PubMed]

17. Protesescu, L.; Yakunin, S.; Bodnarchuk, M.I.; Krieg, F.; Caputo, R.; Hendon, C.H.; Yang, R.X.; Walsh, A.; Kovalenko, M.V. Nanocrystals of Cesium Lead Halide Perovskites ( $\mathrm{CsPb}_{3}, \mathrm{X}=\mathrm{Cl}, \mathrm{Br}$, and I): Novel Optoelectronic Materials Showing Bright Emission with Wide Color Gamut. Nano Lett. 2015, 15, 3692-3696. [CrossRef] [PubMed]

18. Cho, H.; Jeong, S.H.; Park, M.H.; Kim, Y.H.; Wolf, C.; Lee, C.L.; Heo, J.H.; Sadhanala, A.; Myoung, N.; Yoo, S.; et al. Overcoming the Electroluminescence Efficiency Limitations of Perovskite Light-Emitting Diodes. Science 2015, 350, 1222-1225. [CrossRef] [PubMed]

19. Ling, Y.; Yuan, Z.; Tian, Y.; Wang, X.; Wang, J.C.; Xin, Y.; Hanson, K.; Ma, B.; Gao, H. Bright Light-Emitting Diodes Based on Organometal Halide Perovskite Nanoplatelets. Adv. Mater. 2016, 28, 305-311. [CrossRef] [PubMed]

20. Wang, Y.; Li, X.; Song, J.; Xiao, L.; Zeng, H.; Sun, H. All-Inorganic Colloidal Perovskite Quantum Dots: A New Class of Lasing Materials with Favorable Characteristics. Adv. Mater. 2015, 27, 7101-7108. [CrossRef] [PubMed]

21. Wu, K.; Liang, G.; Shang, Q.; Ren, Y.; Kong, D.; Lian, T. Ultrafast Interfacial Electron and Hole Transfer from $\mathrm{CsPbBr}_{3}$ Perovskite Quantum Dots. J. Am. Chem. Soc. 2015, 137, 12792-12795. [CrossRef] [PubMed]

22. Makarov, N.S.; Guo, S.; Isaienko, O.; Liu, W.; Robel, I.; Klimov, V.I. Spectral and Dynamical Properties of Single Excitons, Biexcitons, and Trions in Cesium-Lead-Halide Perovskite Quantum Dots. Nano Lett. 2016, 16, 2349-2362. [CrossRef] [PubMed]

23. Jin, X.; Zhang, X.; Fang, H.; Deng, W.; Xu, X.; Jie, J.; Zhang, X. Facile Assembly of High-Quality Organic-Inorganic Hybrid Perovskite Quantum Dot Thin Films for Bright Light-Emitting Diodes. Adv. Funct. Mater. 2018, 28, 1705189. [CrossRef]

24. Pan, J.; Shang, Y.; Yin, J.; De Bastiani, M.; Peng, W.; Dursun, I.; Sinatra, L.; El-Zohry, A.M.; Hedhili, M.N.; Emwas, A.; et al. Bidentate Ligand-Passivated $\mathrm{CsPbI}_{3}$ Perovskite Nanocrystals for Stable Near-Unity Photoluminescence Quantum Yield and Efficient Red Light-Emitting Diodes. J. Am. Chem. Soc. 2018, 140, 562-565. [CrossRef] [PubMed] 
25. Zhao, L.; Yeh, Y.; Tran, N.L.; Wu, F.; Xiao, Z.; Kerner, R.A.; Lin, Y.L.; Scholes, G.D.; Yao, N.; Rand, B.P. In Situ Preparation of Metal Halide Perovskite Nanocrystal Thin Films for Improved Light-Emitting Devices. ACS Nano 2017, 11, 3957-3964. [CrossRef] [PubMed]

26. Yang, X.; Zhang, X.; Deng, J.; Chu, Z.; Jiang, Q.; Meng, J.; Wang, P.; Zhang, L.; Yin, Z.; You, J. Efficient Green Light-Emitting Diodes based on Quasi-Two-Dimensional Composition and Phase Engineered Perovskite with Surface Passivation. Nat. Commun. 2018, 9, 570. [CrossRef] [PubMed]

27. Sung, J.; Choi, Y.S.; Kang, S.J.; Cho, S.H.; Lee, T.; Park, C. AC Field-Induced Polymer Electroluminescence with Single Wall Carbon Nanotubes. Nano Lett. 2011, 11, 966-972. [CrossRef] [PubMed]

28. Wang, Z.; Chen, Y.; Li, P.; Hao, X.; Liu, J.; Huang, R.; Li, Y. Flexible Graphene-Based Electroluminescent Devices. ACS Nano 2011, 5, 7149-7154. [CrossRef] [PubMed]

29. Cho, S.H.; Sung, J.; Hwang, I.; Kim, R.H.; Choi, Y.S.; Jo, S.S.; Lee, T.W.; Park, C. High Performance AC Electroluminescence from Colloidal Quantum Dot Hybrids. Adv. Mater. 2012, 24, 4540-4546. [CrossRef] [PubMed]

30. Perumal, A.; Fröbel, M.; Gorantla, S.; Gemming, T.; Lüssem, B.; Eckert, J.; Leo, K. Novel Approach for Alternating Current (AC)-Driven Organic Light-Emitting Devices. Adv. Funct. Mater. 2012, 22, 210-217. [CrossRef]

31. Mu, W.; Zhang, P.; Xu, J.; Sun, S.; Xu, J.; Li, W.; Chen, K. Direct-Current and Alternating-Current Driving Si Quantum Dots-Based Light Emitting Device. IEEE J. Sel. Top. Quantum Electron. 2014, 20, $206-211$.

32. Liu, J.; Sheng, X.; Wu, Y.; Li, D.; Bao, J.; Ji, Y.; Lin, Z.; Xu, X.; Yu, L.; Xu, J.; et al. All-Inorganic Perovskite Quantum Dots/p-Si Heterojunction Light-Emitting Diodes under DC and AC Driving Modes. Adv. Opt. Mater. 2018, 6, 1700897. [CrossRef]

33. Liu, J.; Zhang, X.; Ji, Y.; Sheng, X.; Ma, H.; Xu, X.; Yu, L.; Xu, J.; Chen, K. Rational Energy Band Alignment and $\mathrm{Au}$ Nanoparticles in Surface Plasmon Enhanced Si-Based Perovskite Quantum Dot Light-Emitting Diodes. Adv. Opt. Mater. 2018, 6, 1800693. [CrossRef]

34. Swarnkar, A.; Marshall, A.R.; Sanehira, E.M.; Chernomordik, B.D.; Moore, D.T.; Christians, J.A.; Chakrabarti, T.; Luther, J.M. Quantum Dot-Induced Phase Stabilization of a-CsPbI ${ }_{3}$ Perovskite for High-efficiency Photovoltaics. Science 2016, 354, 92-95. [CrossRef] [PubMed]

35. Zhang, L.; Yang, X.; Jiang, Q.; Wang, P.; Yin, Z.; Zhang, X.; Tan, H.; Yang, Y.M.; Wei, M.; Sutherland, B.R.; et al. Ultra-bright and Highly Efficient Inorganic Based Perovskite Light-Emitting Diodes. Nat. Commun. 2017, 8, 15640. [CrossRef] [PubMed] 